\title{
T stage prediction of colorectal tumor based on multiparametric functional images
}

\author{
Yafang Dou ${ }^{1 *}$, Xuefeng Tang ${ }^{1 *}$, Yingying Liu ${ }^{2}$, Zhigang Gong ${ }^{1}$ \\ ${ }^{1}$ Department of Radiology, Affiliated Shuguang Hospital, Shanghai TCM University, Shanghai 201203, China; ${ }^{2}$ Institutes of Biomedical Sciences, \\ Fudan University, Shanghai 200433, China \\ Contributions: (I) Conception and design: Y Dou, X Tang; (II) Administrative support: Z Gong; (III) Provision of study materials: All authors; \\ (IV) Collection and assembly of data: All authors; (V) Data analysis and interpretation: Y Dou, X Tang; (VI) Manuscript writing: All authors; \\ (VII) Final approval of manuscript: All authors. \\ "These authors contributed equally to this work. \\ Correspondence to: Zhigang Gong. Department of Radiology, Affiliated Shuguang Hospital, Shanghai TCM University, Shanghai 201203, China. \\ Email: ericgongzg@aliyun.com; Yingying Liu. Institutes of Biomedical Sciences, Fudan University, Shanghai 200433, China. Email: liuyychina@hotmail.com.
}

Background: Recent studies have shown radiomics parameters of functional imaging have predictive values in many diseases. This study was to investigate the value of radiomics parameters of both computed tomography (CT) and magnetic resonance imaging (MRI) in predicting T stage of colorectal cancer (CRC).

Methods: Imaging findings of CT and MRI (both cT1-W and T2-W) and clinical information were collected from 29 patients. A total of 330 radiomics parameters were computed from manually annotated medical images, and a lasso regression model with 10 -fold cross validation was employed to predict the $\mathrm{T}$ stage with radiomics parameters.

Results: The lasso regression model showed good performance with area under the curve (AUC) of 0.85 . A total of three parameters from MRI were used in this model, while no CT findings were included in this model. The 3 selected parameters were from first-order parameters' group, which include energy and totalenergy from both cT1-W and T2-W. These parameters indicate the magnitude of pixels in the medical images.

Conclusions: This study indicates that some radiomics parameters of functional images have predictive values in T staging of CRC. Also, MRI may be more valuable than CT based on the image findings with lasso regression.

Keywords: Colorectal tumor; T staging; multiparametric functional imaging; regression

Submitted Aug 19, 2019. Accepted for publication Nov 11, 2019.

doi: $10.21037 /$ tcr.2019.11.41

View this article at: http://dx.doi.org/10.21037/tcr.2019.11.41

\section{Introduction}

Numerous studies have shown that the differentiation of malignant tumor is an important prognostic factor (1). In the tumor staging [including colorectal cancer (CRC)], a two-tiered classification system of histologic grade (low grade $=$ well and moderate differentiation; high grade $=$ poor differentiation) is recommended because it has favorable reproducibility and prognostic significance. In addition, there is evidence showing that the risk to relapse is higher for high-grade CRC after tumor resection, which results in poor prognosis (2). It has been shown that pre-operative radiotherapy and/or chemoradiotherapy may improve the local control and disease-free survival in high-risk CRC patients (1). However, neoadjuvant therapies may still cause serious side effects (1). Thus, the differentiation of high-grade CRC from low-grade CRC will be beneficial to maximize the benefit and avoid unnecessary side effects of pre-operatively excessive treatment, helpful to identify patients with high risk for recurrence before treatment and crucial for the individualized therapy, which hence improves outcomes.

The histologic grade determined by pathologic assessment of biopsy samples may offer some information 
of tumor differentiation. However, most tumors are spatially heterogeneous, and there may be discrepancy in the histologic grade between biopsy samples and surgical specimens $(1,3)$. As an invasive examination, colonoscopic biopsy also has complications such as bleeding, colonic perforation and abdominal pain (1).

Great progress has been achieved in the non-invasive diagnostic imaging techniques for CRC in the past decades. Computed tomography (CT) and magnetic resonance imaging (MRI) have been widely used in the preoperative staging of CRC (4-7). Nevertheless, the sensitivity and specificity of them are largely dependent on the classical colorectal morphologic evaluation. In a study of Kim et al. (3), the characteristic CT findings on contrast-enhanced CT were employed to discriminate poorly differentiated CRC from well or moderately differentiated CRC, and their results showed the sensitivity and specificity were $88 \%$ and $70 \%$, respectively, but the subjective assessment of these features has a low reproducibility due to the significant intra- and interobserver variability.

Thus, it is imperative to develop functional primary local staging assessment method with advanced sequences, rather than the method relying only on the morphologic features. A radiomics signature has been developed to assist the differentiation of high-grade from low-grade CRC before treatment and studies have confirmed it as a complementary approach. The radiomics signature, as a non-invasive and quantitative tool to predict the histologic grade before surgery, plays a vital role in the individualized therapy and the improvement of outcomes in CRC patients. Huang et al. (1) reported the colonoscopic biopsy has limited value in the evaluation of histologic grade of CRC, because the overall accuracy was only 0.447 , which was significantly lower than that of radiomics signature (0.775 in training dataset and 0.743 in validation dataset). In addition, some studies have revealed that the combined analysis of multiple features as a radiomics signature may be helpful for the evaluation of correlation between radiomics signature and histologic grade of CRC. Huang et al. (1) developed a radiomics signature and confirmed it was a complementary tool that helps to differentiate high-grade from low-grade CRC preoperatively which is beneficial for the personalized treatment. However, MRI was not included in their study. MRI has been widely accepted as a tool for the pre-treatment evaluation of CRC, especially in the local staging. The key sequences for $\mathrm{T}$ staging are high resolution T2-weighted turbo spin-echo (TSE) images acquired in different planes, planned according to the axis and orientation of the tumor. Generally, MRI has a high sensitivity for local staging of the primary tumor, especially when $\mathrm{T}$ staging is considered. MRI has the potential to evaluate and predict circumferential resection margin (CRM) and extramural invasion (including venous invasion) with a high accuracy. In addition, studies have shown that it is a reproducible technique because its specificity is as high as $92 \%$ in predicting the negative CRM, the depth of invasion beyond muscularis propria and the relationship of the tumor with the CRM (7-10). In a study of Sun et al. (11), results showed pre-treatment T2WI radiomics could be used to characterize the histopathological features of rectal cancer, which suggests that tumor-derived radiological features may be employed for $\mathrm{T}$ staging.

In addition, the contrast-enhanced CT has also been frequently used in the management of CRC, and thus CTbased radiomics signature has better popularization and application in differentiating high-grade from low-grade CRC.

To the best of our knowledge, no study has been conducted to investigate the correlation between radiomics signature and histologic grade of CRC based on both CT and MRI findings. This study was undertaken to develop and validate a tumor stage prediction model based on the findings from functional imaging including $\mathrm{CT}$ and MRI (including cT1-W and $\mathrm{T} 2-\mathrm{W}$ images). Lasso regression model with 10 -fold cross-validation was employed on the set of image parameters to predict the T stage of CRC.

\section{Methods}

\section{Patients and data acquisition}

Clinical records and functional images of 41 patients were retrieved in this study under the ethical board approval of Shuguang hospital affiliated to Shanghai Traditional Chinese Medical University from June 2018 to April 2019. Patients' written consent were not needed because this study is a retrospective study, and both CT and MRI were performed as regular examinations in our institution. This study aims to investigate the association between the parameters of functional images and $\mathrm{T}$ stage. Thus the $\mathrm{T}$ stage of the involved patients must be confirmed based on pathology diagnosis. To study the parameters of both MRI and CT images, the contrast-enhanced CT images, MR cT1-w and MR T2-w images of each patient must be available. Based on the two criteria, 29 were selected out of the 41 patients in this study. Among them, 6 was confirmed 
Table 1 Characteristics of patients-CT, MR

\begin{tabular}{lll}
\hline Characteristics & Early-stage CRC & Late-stage CRC \\
\hline Age (year) & $66.67 \pm 6.80$ & $70.83 \pm 7.43$ \\
Male & $5(5 / 29)$ & $1(1 / 1)$ \\
Female & $1(1 / 29)$ & $7(7 / 29)$ \\
Tumor pathologic types & Chorionic tubular adenoma with intraepithelial neoplasia 2 (2/29) & T3: Adenocarcinoma 21 (21/29); \\
& Adenocarcinoma 1 (1/29) & Mucinous adenocarcinoma 1 (1/29); \\
& Adenocarcinoma 3 (3/29) & T4: 0 \\
Size of tumor (mm) & & $62.69 \pm 34.22$ \\
Long diameter & $42.57 \pm 12.43$ & $24.96 \pm 14.19$ \\
Short diameter & $20.6 \pm 7.27$ & carcinoma 1 (1/29) \\
\hline
\end{tabular}

MR, magnetic resonance; CT, computed tomography; CRC, colorectal cancer.

with early-stage tumor ( $\mathrm{T}$ stage: Tis, 1 and 2), and 23 were confirmed with late-stage tumor (T stage: 3,4$)$. The characteristics of the patients are shown in Table 1.

Both the patients' CT scans and MRI scans were performed on the same day. All the patients CT scans were performed with a DECT scanner (SOMATOM Force; Siemens Healthineers, Forchheim, Germany), and all the MRI scans were performed on a 3.0-T system (uMR 780, united-imaging healthcare, China). A set of preparations were performed before the examinations. All patients were instructed to undergo the enema with water (about $500-1,000 \mathrm{~mL}$ ) on the night before the DECT examination. On the day of the DECT examination, the patient's colon or rectum was not distended. The per-colonic or rectal contrast was setup according to the tumor location. In order to minimize the artifacts caused by respiratory motion, all the patients were asked to breathe quietly during their perfusion CT (PCT) examination.

In order to select the scan range for the PCT scan, a non-contrast scan of the entire abdominal cavity was performed at first, which covers the region from the lung base to the lower margin of the symphysis pubis. A VPCT scan was followed afterwards. Contrast injection was performed 5 seconds' prior to the VPCT scan, which includes a $33 \mathrm{~mL}$ of a nonionic iodinated contrast agent (350 mg/mL, Bonorex ${ }^{\circledR}$ 350; Central Medical Service) and a $33 \mathrm{~mL}$ saline flush via an antecubital vein. The scan coverage for PCT was setup to be $17.6 \mathrm{~cm}$. The cycle time was 1.5 seconds. And a total of 26 scans were acquired during 40 seconds' scanning. The detector collimation of the PCT was $48 \times 1.2 \mathrm{~mm}$. The gantry rotation time was
0.32 seconds with a tube current-time product of $60 \mathrm{mAs}$ at $80 \mathrm{kVp}$. A $3 \mathrm{~mm}$ increment was used to reconstruct the PCT images with a $5 \mathrm{~mm}$ thickness. DECT scan was performed after acquiring the PCT images. An additional contrast was injected 30 seconds before DECT scan, which include $1.2 \mathrm{~mL}$ of per kilogram of body weight and a $25 \mathrm{~mL}$ saline flush via an antecubital vein. The contrast was injected at a $3 \mathrm{~mL} / \mathrm{s}$ flow rate. The tube voltage of 100 and Sn150 kVps and respective ref. tube current time products of 180 and $90 \mathrm{mAs}$ were used. The detector collimation of the DECT scan was setup to be $128 \times 0.6 \mathrm{~mm}$. And gantry rotation time was 0.5 seconds. The image reconstruction was performed with $1.5 \mathrm{~mm}$ thickness at $1 \mathrm{~mm}$ increments.

After the acquisition of CT images, MRI was performed on the same day. Prior to the MRI scan, a $20 \mathrm{mg}$ of phloroglucinol was injected to each patient intramuscularly, in order to minimize intestinal peristalsis. The patients didn't perform colorectal distension or antispasmodic medication. Routine $3 \mathrm{~mm}$ MRI scan was performed with no inter-slice gap, which include oblique sagittal and axial T2-weighted (T2-w), axial T1-weighted (T1-w) and contrast-enhanced T1-weighted (cT1-w) images. A 3D dynamic sequence was used to perform DCE-MRI. High spatial and temporal resolution was achieved with time-resolved technique based on inter-leaved stochastic trajectories (3D-QUICK). After DCE-MRI, gadopentetate dimeglumine $(0.1 \mathrm{mmol} / \mathrm{kg}$ body weight, at a rate of $3.0 \mathrm{~mL} / \mathrm{s})$ and saline flush $(25 \mathrm{~mL})$ were injected to the patients. Thereafter, the axial images were obtained in 25 dynamic phases with $3 \mathrm{~mm}$ thickness, as well as the postcontrast coronal 3D T1W images with $3.0 \mathrm{~mm}$ thickness at 
Table 2 MRI parameters

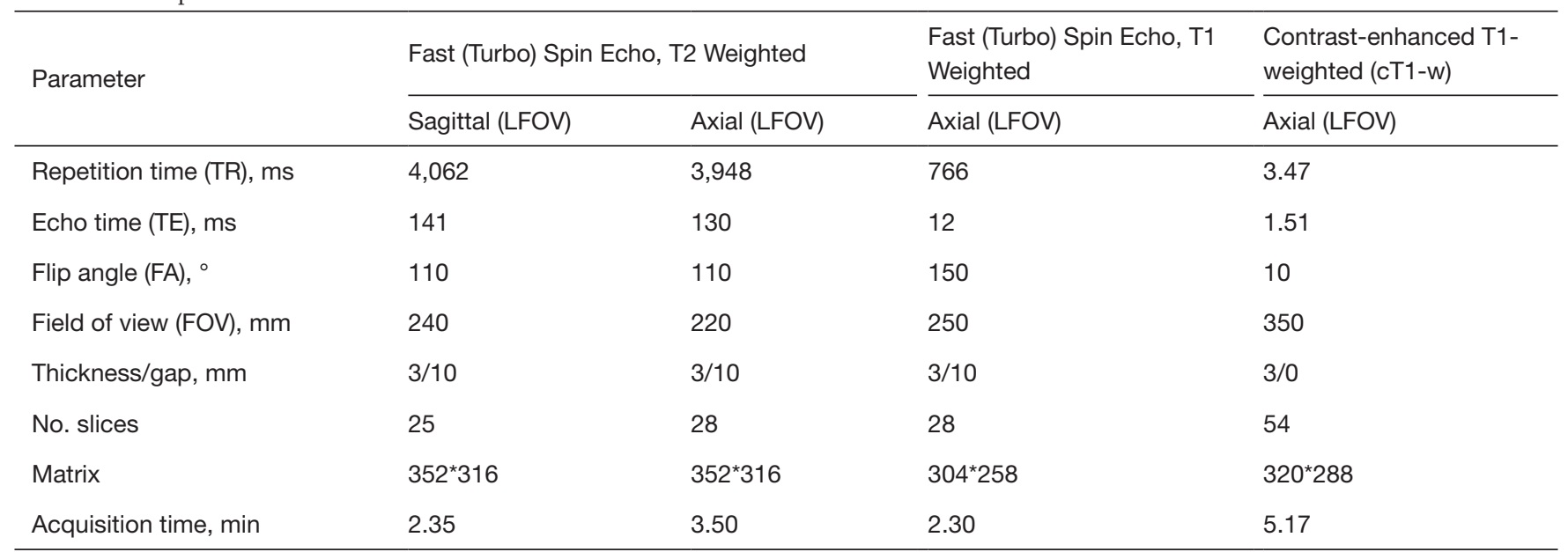

MRI, magnetic resonance imaging.

$10 \mathrm{~mm}$ interval. The details of involved parameters in the colorectal MR protocol and image acquisition process are presented in Table 2.

\section{Parameters}

In order to compute the image parameters from the tumor region, on each slice of the functional images, the tumor region was manually labelled by experienced radiologists using a free software named ITK-snap (9). Examples of the labelled regions are shown in Figure 1. Each tumor was labelled on multiple slices of the functional image.

Radiomics parameters were extracted from 3D volumes constructed from the labelled tumor region in each slice in many previous studies. However, the colorectal tumor has irregular shapes, which varies largely among slices and often occupies many slices in one image. Thus we believe that computing parameters from $3 \mathrm{D}$ volume will introduce additional variations and noises due to the $3 \mathrm{D}$ volume reconstruction from $2 \mathrm{D}$ regions. Instead, in this study, parameters were computed from slices without forming $3 \mathrm{D}$ volumes. The parameters were averaged over all slices of each patient and used for further analysis. The maximal values and the minimal values were removed from the averaging. Therefore, in order to compute the average values, the patients' images must contain at least 3 slices. From each of the CT contrast-enhanced image, MR cT1-w image and T2-w image, 110 image parameters were computed using an open-source Python package PyRadiomics (12). Therefore, a total of 330 image parameters were obtained for each patient.

\section{Analysis}

A classical regression analysis method named least absolute shrinkage and selection operation (Lasso) regression was used to build a prediction model of tumor $\mathrm{T}$ staging based on the pairs of image parameters and patients' $\mathrm{T}$ stages. A 10 -fold cross-validation was applied. The regression method automatically assign non-zero weights to part of the parameters during the model training. Thus only parameters with meanings for the model were selected. The model was trained on the pairs of the computed image parameters and the patients' $\mathrm{T}$ stage.

\section{Results}

The patients were divided into non-metastatic group and metastatic group based on their tumor stages. Nonmetastatic group refers to the early stage patients, and metastatic group refers to the late stage patients. The parameters were computed from the labelled tumor regions and averaged over slices for each patient. As at least 3 slices were needed for the computation of the average value, 3 patients were excluded due to that the number of labelled slices were fewer than 3 . A total of 26 patients were analyzed. Among them, 4 were with early-stage CRC and 22 were with late-stage CRC.

Based on 10-fold cross-validation, three parameters with non-zeros coefficients were selected by the lasso regression model (see Table 3). It can be seen that all the selected parameters are first-order parameters. Among them, two are parameters of MR cT1-w images, the other one is 

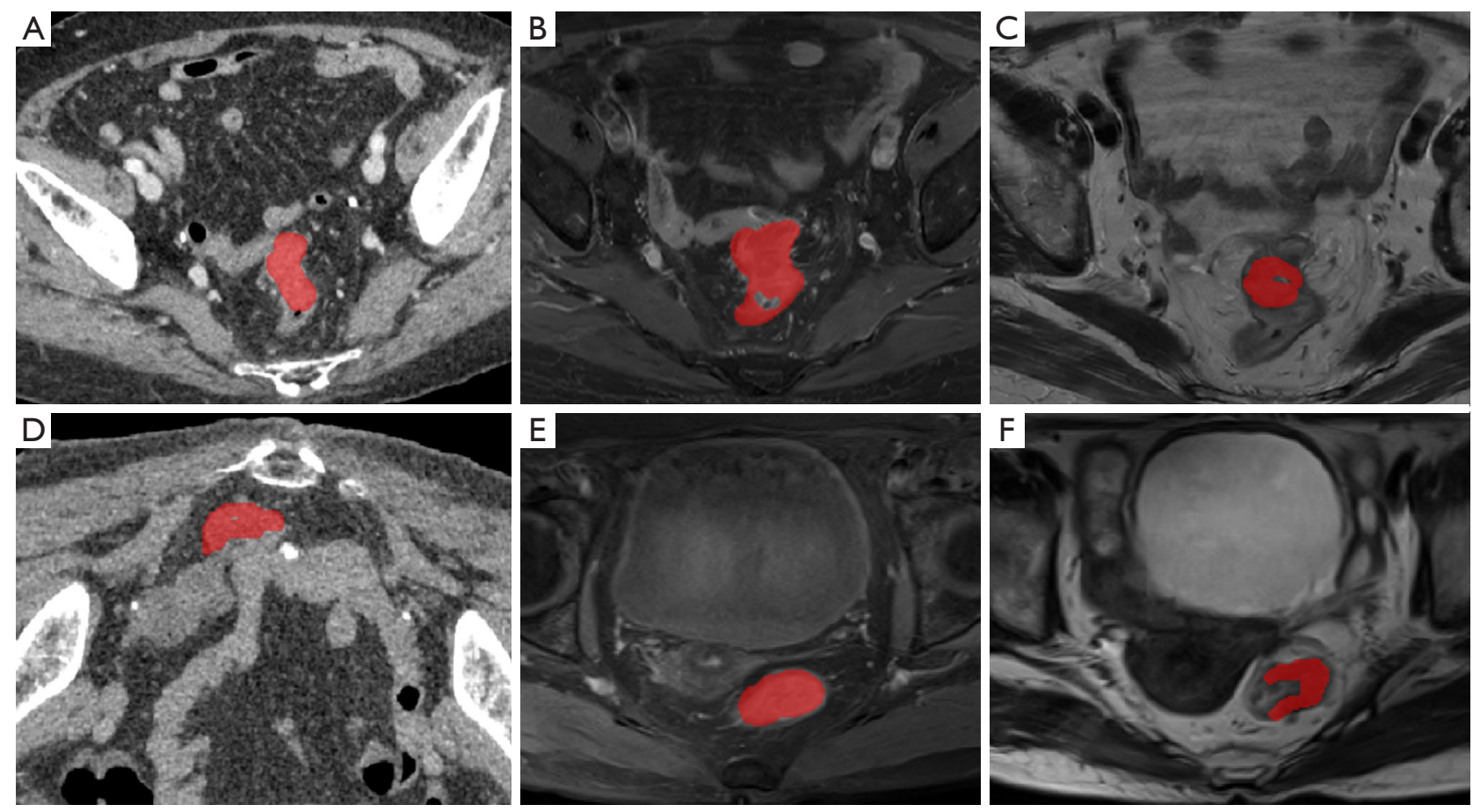

Figure 1 Example of labelled tumor regions. (A) Example of metastical tumor in CT image; (B) example of metastical tumor in MR T1 contrast image; (C) example of metastical tumor in MR T2 image; (D) example of benign tumor in CT image; (E) example of benign tumor in MR T1 contrast image; (F) example of benign tumor in MR T2 image. MR, magnetic resonance; CT, computed tomography.

Table 3 Selected image parameters with non-zero coefficients based on lasso regression

\begin{tabular}{lccc}
\hline $\begin{array}{l}\text { CT image } \\
\text { parameters }\end{array}$ & $\begin{array}{c}\text { T1 contrast image } \\
\text { parameters }\end{array}$ & $\begin{array}{c}\text { T2 image } \\
\text { parameters }\end{array}$ \\
\hline & $\begin{array}{c}\text { First-order: } \\
\text { energy }\end{array}$ & $\begin{array}{c}\text { First-order: } \\
\text { total energy }\end{array}$ & $\begin{array}{c}\text { First-order: } \\
\text { total energy }\end{array}$ \\
\hline
\end{tabular}

parameters of MR T2-images. None of the parameters of CT images is selected. The AUC obtained by the lasso regression model is 0.85 (see Figure 2).

\section{Discussion}

CT and MRI are the two main image resources for preoperative diagnosis of patients with colorectal tumor, which are often involved in their routine examinations. Previous studies have shown the predictive values of either CT or MRI on colorectal tumor (13-18). However, no study had used both of them. This study aimed to investigate the predictive values

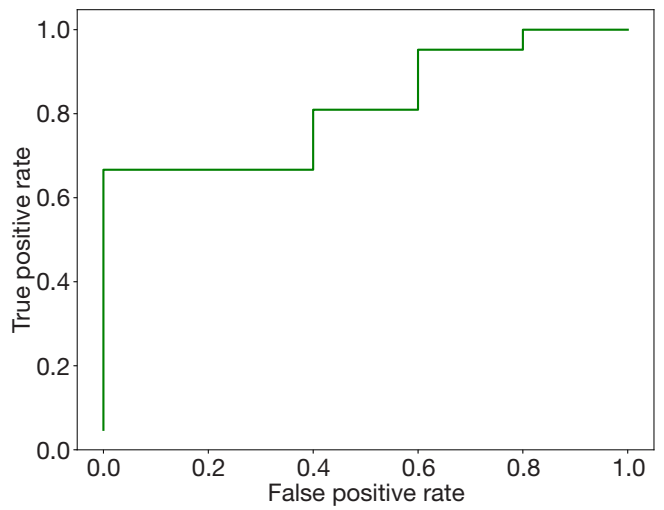

Figure 2 ROC curve of the Lasso regression model (AUC $=0.85$ ). ROC, receiver operating characteristic curve; AUC, area under the curve.

of the Radiomics parameters of both CT and MR images in colorectal tumor $\mathrm{T}$ staging. A total of 330 image parameters were computed from CT image, MR cT1-W image and MR $\mathrm{T} 2-\mathrm{W}$ image for each patient with confirmed $\mathrm{T}$ stage based on pathology. A prediction model was constructed based on the pairs of the parameters and the $\mathrm{T}$ stage using classical lasso 
regression with 10 -fold cross-validation.

It can be seen from Figure 2 that the AUC of the prediction model is 0.85 , which demonstrate the pontential predictive value of the radiomics parameters. The selected parameter consists of one energy parameters and two total energy parameters. Based on definition (19), first-order statistics describe the distribution of the image intensities. Both energy and total energy measure the magnitude of the pixels in the image. Larger values imply a greater sum of the squares of the pixel values. This demonstrates that the magnitude of the pixels in the MR images have the potential to be used in colorectal tumor staging.

Moreover, it can be concluded that all the three selected parameters are first-order parameters from MR images (see Table 3), while no parameter is selected from CT image. This indicate that MR images may have more value thant CT images in tumor staging prediction using modern computer-aided method. In the present clinical process, CT is a more widely applied technique than MRI in the preoperative tumor staging due to the low cost and fast speed. In this study, by comparing CT with MRI, it reveals that MRI may be more valuable than CT. In clinical practices, many factors must be considered during pre-operative diagnosis and planning, for example the cost, scan time, radiation and predictive value. Thus, whether CT or MRI is to be used is related to the on-site situations.

\section{Conclusions}

This study investigated the predictive value of both CT and MR images in the evaluation of colorectal cancer staging. A lasso regression model was used to construct the prediction model based on Radiomics parameters of functional images. Based on the results, it is demonstrated that three parameters of MR (cT1-w and T2-w) images may have predictive value in the colorectal tumor staging evaluation. The number of patients included in our study is limited, therefore, CT and MR images of each patient are needed for evaluation. Our further study will focus on collecting more patients' data and validate the proposed model.

\section{Acknowledgments}

Funding: None.

\section{Footnote}

Conflicts of Interest: The authors have completed the
ICMJE uniform disclosure form (available at http://dx.doi. org/10.21037/tcr.2019.11.41). The authors have no conflicts of interest to declare.

Ethical Statement: The authors are accountable for all aspects of the work in ensuring that questions related to the accuracy or integrity of any part of the work are appropriately investigated and resolved. The study was conducted in accordance with the Declaration of Helsinki (as revised in 2013). This study had been approved by the Ethics Committee of Affiliated Shuguang Hospital, Shanghai TCM University (2019-687-42). Patients' written consent were not needed because this study is a retrospective study.

Open Access Statement: This is an Open Access article distributed in accordance with the Creative Commons Attribution-NonCommercial-NoDerivs 4.0 International License (CC BY-NC-ND 4.0), which permits the noncommercial replication and distribution of the article with the strict proviso that no changes or edits are made and the original work is properly cited (including links to both the formal publication through the relevant DOI and the license). See: https://creativecommons.org/licenses/by-nc$\mathrm{nd} / 4.0 /$.

\section{References}

1. Huang X, Cheng Z, Huang Y, et al. CT-based Radiomics Signature to Discriminate High-grade From Low-grade Colorectal Adenocarcinoma. Acad Radiol 2018;25:1285-97.

2. Bockelman C, Engelmann BE, Kaprio T, et al. Risk of recurrence in patients with colon cancer stage II and III: a systematic review and meta-analysis of recent literature. Acta Oncol 2015;54:5-16.

3. Kim JE, Lee JM, Baek JH, et al. Differentiation of poorly differentiated colorectal adenocarcinomas from well- or moderately differentiated colorectal adenocarcinomas at contrast-enhanced multidetector CT. Abdom Imaging 2015;40:1-10.

4. Liang C, Huang Y, He L, et al. The development and validation of a CT-based radiomics signature for the preoperative discrimination of stage I-II and stage III-IV colorectal cancer. Oncotarget 2016;7:31401-12.

5. Wen Z, Chen Y, Yang X, et al. Application of magnetic resonance diffusion kurtosis imaging for distinguishing histopathologic subtypes and grades of rectal carcinoma. Cancer Imaging 2019;19:8.

6. He B, Ji T, Zhang H, et al. MRI-based radiomics signature 
for tumor grading of rectal carcinoma using random forest model. J Cell Physiol 2019;234:20501-9.

7. Gurses B, Boge M, Altinmakas E, et al. Multiparametric MRI in rectal cancer. Diagn Interv Radiol 2019;25:175-82.

8. Costa-Silva L, Brown G. Magnetic resonance imaging of rectal cancer. Magn Reson Imaging Clin N Am 2013;21:385-408.

9. Hotker AM, Garcia-Aguilar J, Gollub MJ. Multiparametric MRI of rectal cancer in the assessment of response to therapy: a systematic review. Dis Colon Rectum 2014;57:790-9.

10. Sun Y, Li J, Shen L, et al. Predictive value of MRIdetected extramural vascular invasion in stage T3 rectal cancer patients before neoadjuvant chemoradiation. Diagn Interv Radiol 2018;24:128-34.

11. Sun Y, Hu P, Wang J, et al. Radiomic features of pretreatment MRI could identify $T$ stage in patients with rectal cancer: Preliminary findings. J Magn Reson Imaging 2018. [Epub ahead of print].

12. Yushkevich PA, Piven J, Hazlett HC, et al. Userguided 3D active contour segmentation of anatomical structures: significantly improved efficiency and reliability. Neuroimage 2006;31:1116-28.

13. De Cecco CN, Ciolina M, Caruso D, et al. Performance of diffusion-weighted imaging, perfusion imaging, and texture analysis in predicting tumoral response to

Cite this article as: Dou Y, Tang X, Liu Y, Gong Z. T stage prediction of colorectal tumor based on multiparametric functional images. Transl Cancer Res 2020;9(2):522-528. doi: 10.21037/tcr.2019.11.41 neoadjuvant chemoradiotherapy in rectal cancer patients studied with 3T MR: initial experience. Abdom Radiol (NY) 2016;41:1728-35.

14. Morani AC, Elsayes KM, Liu PS, et al. Abdominal applications of diffusion-weighted magnetic resonance imaging: Where do we stand. World J Radiol 2013;5:68-80.

15. Nie K, Shi L, Chen Q, et al. Rectal Cancer: Assessment of Neoadjuvant Chemoradiation Outcome based on Radiomics of Multiparametric MRI. Clin Cancer Res 2016;22:5256-64.

16. Petrillo A, Fusco R, Granata V, et al. MR imaging perfusion and diffusion analysis to assess preoperative Short Course Radiotherapy response in locally advanced rectal cancer: Standardized Index of Shape by DCE-MRI and intravoxel incoherent motion-derived parameters by DW-MRI. Med Oncol 2017;34:198.

17. Pham TT, Liney G, Wong K, et al. Study protocol: multiparametric magnetic resonance imaging for therapeutic response prediction in rectal cancer. BMC Cancer 2017;17:465.

18. Xu Y, Xu Q, Sun H, et al. Could IVIM and ADC help in predicting the KRAS status in patients with rectal cancer? Eur Radiol 2018;28:3059-65.

19. van Griethuysen JJM, Fedorov A, Parmar C, et al. Computational Radiomics System to Decode the Radiographic Phenotype. Cancer Res 2017;77:e104-7. 